\title{
Fine mapping and characterization of a novel dwarf and narrow-leaf mutant $d n l l$ in rice
}

\author{
X.J. Wei ${ }^{1 *}$, S.Q. Tang ${ }^{1 *}$, G.N. Shao ${ }^{1}$, M.L. Chen ${ }^{1}$, Y.C. Hu ${ }^{2}$ and P.S. Hu${ }^{1}$ \\ ${ }^{1}$ Chinese National Center for Rice Improvement, \\ State Key Laboratory of Rice Biology, China National Rice Research Institute, \\ Hangzhou, China \\ ${ }^{2}$ Hunan Agricultural University, Changsha, China \\ *These authors contributed equally to this study. \\ Corresponding author: P.S. Hu \\ E-mail: hupeisong@yahoo.com.cn / riceh@caas.net.cn
}

Genet. Mol. Res. 12 (3): 3845-3855 (2013)

Received December 17, 2012

Accepted July 23, 2013

Published September 23, 2013

DOI http://dx.doi.org/10.4238/2013.September.23.2

\begin{abstract}
Plant height is one of the most important agronomic traits of rice (Oryza sativa). Dwarf mutants are ideal materials for research on the mechanisms of regulation of rice plant height. We examined a new dwarf and narrow-leaf mutant $d n l 1$. Phenotypic analysis showed that the $d n l 1$ mutant has a thinner culm and more tillers, but the number of grains per panicle, the seed setting rate and the grain weight of $d n l 1$ mutant were found to be significantly lower than in the wild-type. Based on scanning electron microscopic observations, the number of cells in the y-axis in internodes was significantly lower than in the wild-type. In phytohormone induction experiments, $d n l 1$ was gibberellic acidinsensitive. The expression of some genes involved in the gibberellins metabolic pathways was affected in the $d n l 1$ mutant, based on the realtime PCR analysis, suggesting that the $d n l 1$ gene likely plays a role in gibberellin metabolic pathways. Genetic analysis showed that the dwarf and narrow leaf phenotype is controlled by a novel single recessive gene, here referred to as the dwarf and narrow leaf $1(d n l 1)$, which is located within the region between markers Ind12-11 and RM8214 on the short arm of chromosome 12. By means of fine-mapping strategy,
\end{abstract}


the $d n l 1$ gene was localized within an interval of $285.75 \mathrm{~kb}$ physical distance. These results will be useful for dnll gene cloning and to improve our understanding of the molecular mechanisms involved in the regulation of growth and development of rice.

Key words: Dwarf and narrow leaf; Gibberellic acid-insensitive; Fine mapping; Rice

\section{INTRODUCTION}

The plant height is one of the most important agronomic traits of rice (Oryza sativa). The yield potential of rice has been greatly increased by the so-called rice 'green revolution', which is represented by rice dwarf breeding (Hargrove and Cabanilla, 1979; Khush, 1999). Up to now, the screening and development of rice dwarf sources, genetic analysis, molecular mapping, gene cloning, and molecular mechanism research about rice plant height have led to many great advances (Ikeda et al., 2001; Sasaki et al., 2002, 2003; Hong et al., 2003; Ueguchi-Tanaka et al., 2000, 2005; Tanabe et al., 2005; Arite et al., 2007). However, find new genes for rice plant height and research its genetic mechanisms is still a research focus in rice genetics and genomics.

The dwarf mutants are ideal materials for research on the mechanism for the regulation of rice plant height. Up to the present, about 60 rice dwarf mutants have been reported (Matsuo et al., 1997). Besides having a short plant height, many dwarf mutants also have many other abnormal phenotypes, such as small grains, multiple tillers, narrow or rolled leaves (UeguchiTanaka et al., 2000; Tanabe et al., 2005; Arite et al., 2007; Li et al., 2009). The control of rice plant height is mostly related to the biosynthesis of and responsiveness to phytohormones, such as gibberellic acids (GAs), brassinolide (BL), and strigolactone (Ueguchi-Tanaka et al., 2000; Sasaki et al., 2002, 2003; Hong et al., 2003; Itoh et al., 2004; Tanabe et al., 2005; Arite et al., 2007; Lin et al., 2009). GAs are essential regulators of diverse growth and developmental processes in plants (Davies, 1995). The biosynthesis of GAs has been well characterized (Hedden and Philips, 2000; Sakamoto et al., 2004). Dwarf mutants such as $s d 1, d 18, d 35$ are related to the biosynthesis of GAs. The semidwarf phenotype of $s d 1$ is the result of a deficiency of active GAs in the elongating stem arising from a defective 20-oxidase GA biosynthetic enzyme (Sasaki et al., 2002). The $d 18$ and $d 35$ also are mutants of the GA biosynthetic enzyme genes GA-3 $\beta$-hydroxylase (OsGA3ox2) and ent-kaurene oxidase (OsKO2) (Itoh et al., 2001, 2004). These dwarf mutants always show a low content of active GAs and can be rescued by the application of active GAs such as GA3. However, when the dwarf mutants have a defect in GA responsiveness, such as $d 1$, slr 1 , gidl and gid2, they are insensitive to exogenous GAs (Ikeda et al., 2001; Sasaki et al., 2003; Ueguchi-Tanaka et al., 2000, 2005). The $d 1$ mutant, which is defective in the $\alpha$ subunit of the heterotrimeric G protein $(\mathrm{G} \alpha)$, exhibits dwarf and other GA deficiency phenotypes. The content of endogenous GA in the $d l$ mutant is significantly higher than in the wild-type (Ueguchi-Tanaka et al., 2000). The GID1 gene encodes a soluble GA receptor, the GID2 gene encodes an F-box protein, and the SLR1 gene encodes a DELLA protein. GID1 and GID2 both interact with SLR1, which is a repressor of GA signaling, mediating GA signaling in rice (Sasaki et al., 2003; Ueguchi-Tanaka et al., 2005). However, how plants perceive GA and how the GA signal is transmitted to cause GA-regulated plant growth are still not well known. 
In the present study, the characteristics of a new dwarf and narrow-leaf mutant, dnl1, were studied in detail using morphological and cytological methods. The results of phytohormone induction suggested that $d$ warf and narrow leaf $1(d n l 1)$ is a GA-insensitive mutant. The mutant gene was fine-mapped to a narrow chromosome region. These results are very useful for $D N L 1$ gene cloning and to improve our understanding of the molecular mechanism of the regulation of rice plant height.

\section{MATERIAL AND METHODS}

\section{Plant materials}

The dnll mutant has dwarf, narrow leaf, and thin culm characters, which was obtained from the progeny of tissue culture of a Japonica rice cultivar Asominori. The $\mathrm{F}_{1}$ hybrids and $\mathrm{F}_{2}$ populations derived from the crosses between $d n l l$ mutant and its wild-type cv. Asominori, and another Indica rice cultivar Nanjin11 were used for genetic analysis and fine mapping of the $d n l l$ mutant gene. All materials were grown in the field from 2010 to 2012 in Hangzhou, Zhejiang Province. Sowing was around May 20 and seedlings were transplanted about June 15. Crop management was according to commercial rice production practices.

\section{Scanning electron microscopic observation}

Culms of $d n l 1$ mutant and its wild-type cv. Asominori were harvested 10 days after flowering and fixed in $2.5 \%(\mathrm{v} / \mathrm{v})$ glutaraldehyde. The samples were then post fixed in $2 \%$ $(\mathrm{w} / \mathrm{v}) \mathrm{OsO}_{4}$ for $2 \mathrm{~h}$, dehydrated through an ethanol series, infiltrated, and embedded in butyl methyl methacrylate. The samples were critical-point dried, sputter coated with platinum and examined using a scanning electron microscope (TM-1000, Hitachi).

\section{Genetic analysis and fine mapping of the dnl1 mutant gene}

The $\mathrm{F}_{1}$ hybrids and $\mathrm{F}_{2}$ populations derived from the crosses between $d n l 1$ mutant and its wild-type cv. Asominori, cv. Nanjin 11 were used for genetic analysis. The $\mathrm{F}_{2}$ populations derived from the crosses between the $d n l 1$ mutant and cv. Nanjin11 were used for mapping the $d n l 1$ mutant gene. A total of 901 dwarf and narrow leaf plants were selected from the $\mathrm{F}_{2}$ population derived from the crosses between $d n l l$ mutant and $\mathrm{cv}$. Nanjin11 to fine-map the $d n l l$ mutant gene, using the approach described by Zhang et al. (1994). The molecular markers including SSR and InDel markers used for fine-mapping dnll are shown in Table 1. PCR was performed in a $10-\mu \mathrm{L}$ reaction volume containing $25 \mathrm{ng}$ template DNA, $1.0 \mu \mathrm{L}$ 10X PCR buffer, $0.1 \mathrm{mM}$ dNTP, $0.1 \mu \mathrm{M}$ primer pairs, and $0.1 \mathrm{U}$ Taq DNA polymerase. The amplification protocol included an initial denaturation at $95^{\circ} \mathrm{C}$ for $3 \mathrm{~min}$, followed by 35 cycles of $95^{\circ} \mathrm{C}$ for $30 \mathrm{~s}, 30 \mathrm{~s}$ annealing at $55^{\circ} \mathrm{C}$, and $72^{\circ} \mathrm{C}$ for $30 \mathrm{~s}$, and a final extension step at $72^{\circ} \mathrm{C}$ for $5 \mathrm{~min}$ in a DNA Engine Thermal Cycler. PCR products were separated on a $6 \%$ polyacrylamide gel, and the amplified DNA fragments were silverstained for visualization. 
Table 1. Sequences of primers used for mapping dnll.

\begin{tabular}{lll}
\hline Primer name & Forward $\left(5^{\prime}-3^{\prime}\right)$ & Reverse $\left(5^{\prime}-3^{\prime}\right)$ \\
\hline Ind12-11 & GCCCGCAAGCAGGTCAAGGA & TCACCAGGGAGGAAGAAGA \\
Ind12-14 & TTATCCACATTGTCACATC & GCAGTCAAGTAGCAGTAATA \\
Ind12-16 & AAGAAGACGAAGCCAAAAT & AACCGATACAGTCACAACACT \\
Ind12-2 & GCTTGACCTACGTTTTATTT & CTTCCTCGTGCGTGCTATG \\
Ind12-6 & CACGATGGTCGCCGTAGGC & CCAAGAGCCCCTCGAAGATTTCA \\
Ind12-7 & AGCGACGAGGCCATAGACAT & TGGACGCATCCCGAAATCA \\
Ind12-8 & CGGAGGTTACTGATGTTATG & CTTGAAGATGCCTCTGCTC \\
RM1 1880 & ACCACTAAATAAGCACATAC & GGCATCATACATTAAAATAC \\
\hline
\end{tabular}

\section{GA and BL induction in shoot elongation}

Seeds of the wild-type cv. Asominori and $d n l 1$ were sterilized with $2.5 \% \mathrm{NaClO}$ for $30 \mathrm{~min}$, washed 5 times in sterile distilled water, and incubated at $35^{\circ} \mathrm{C}$ for 2 days. The seeds were then placed on $1 \%$ agar plates containing various concentrations of GA3 and BL, and grown under continuous fluorescent lighting at $30^{\circ} \mathrm{C}$. After 7 days (GA3 induction) or 2 weeks (BL induction), the length of the second leaf sheath of each plant was measured. A total of 30 plants were used for each treatment.

\section{RNA extraction and real-time PCR}

Total RNA from whole 40-day-old seedlings of $d n l l$ mutants and wild-type plants were isolated using an RNA extraction kit following manufacturer instructions (Beijing Dingguo Biotechnology Co. Ltd., http://www.dingguo.com). First-strand cDNA was reverse-transcribed from DNase I-treated RNA with oligo(dT) as the primer. Gene expression was measured by quantitative real-time PCR using the ubiquitin gene (GenBank accession No. AF184280) as internal control. The quantitative real-time PCR primers for genes involved in the GA biosynthesis and metabolic pathways D1, SLR1, GID1, GID2, EUI1, GA20ox2, GA20ox3, GA3ox2, GA2ox1, and GA2ox3 are listed in Table 2. The quantitative real-time PCR was carried out in a total volume of $20 \mu \mathrm{L}$ containing $2 \mu \mathrm{L}$ reverse-transcribed product above, $0.2 \mu \mathrm{M}$ each primer, and 1X SYBR green PCR master mix (TaKaRa Co. Ltd., http://www.takara.com.cn). PCR was performed with a Roche LightCycler 480 (http://www.roche-applied-science.com) using the following program: $95^{\circ} \mathrm{C}$ for $30 \mathrm{~s}$, then 40 cycles of $95^{\circ} \mathrm{C}$ for $5 \mathrm{~s}, 60^{\circ} \mathrm{C}$ for $30 \mathrm{~s}$, and $72^{\circ} \mathrm{C}$ for $30 \mathrm{~s}$. Changes in gene expression were calculated via the $\Delta \Delta \mathrm{CT}$ method.

Table 2. Sequences of primers used for quantitative real-time RT-PCR analysis.

\begin{tabular}{llll}
\hline Genes & Accession No. & Forward (5'-3') & Reverse (5'-3') \\
\hline D1 & D 38232 & AATGCGGTAATCTTTTGTGCTG & AGAGTTCCTTGGTCTCCATCATTC \\
SLR1 & AB030956 & GGTGCGGCCAAGGATCGTCA & GAGGAGCGTGCTCGCCTGTTT \\
GID1 & AB211399 & AGGACAGGGACTGGTACTGGAAGG & TCGTGGTAGTGGACGGTGTTGG \\
GID2 & AB100246 & GTGCGGGAGTGGGCGAACCT & GGCGGATGTAGACAGCGTGGAG \\
EUI1 & AY987039 & CGGGCTTGCTTTGGGAGTGAT & GCGAAGGGATGCTGAAGATGACG \\
GA20ox2 & AB077025 & CGCTGTCGAACGGGAGGTATAAGAG & CATGAGGTCGGCCCAGGTGAAGT \\
GA20ox3 & NM 001065522 & CGAGTTCACGCAGAGGCACTACCG & TTGCTTGATCCAGGCGACGAAGG \\
GA3ox2 & AB 056519 & CCGACGAGTTGCTGAGGTTGT & GCACGAAGGTGAAGAAGCCCGAGT \\
GA2ox1 & AB059416 & CGAGGGCAAGGAGATGTGGG & GTGGGCTGCTGGCTGTGATT \\
GA20x3 & AB092485 & GCGGGTGATGGAGGCGATGT & TTGAGCGGAGCACGGAGACG \\
\hline
\end{tabular}




\section{RESULTS}

\section{Phenotypes of the dnll mutant}

Compared with its wild-type cv. Asominori, the $d n l 1$ mutant showed dwarf, narrow leaf, and thin culm characters throughout growth and development (Figure 1A-D and Table 3 ). The tiller number of $d n l l$ was higher, but the number of grains per panicle and the seed setting rate was significantly lower compared with wild-type (Figure 1B, C, E and Table 3). Otherwise, the grain-filling of the dnll mutant was not sufficient and the grain weight of the dnll mutant was about $42 \%$ lower than that of wild-type, and as a result of that, it had no close spikelet glumes (Figure 1F, G and Table 3). Thus, the DNL1 gene has pleiotropic effects on rice plant height, yield, and plant architecture.
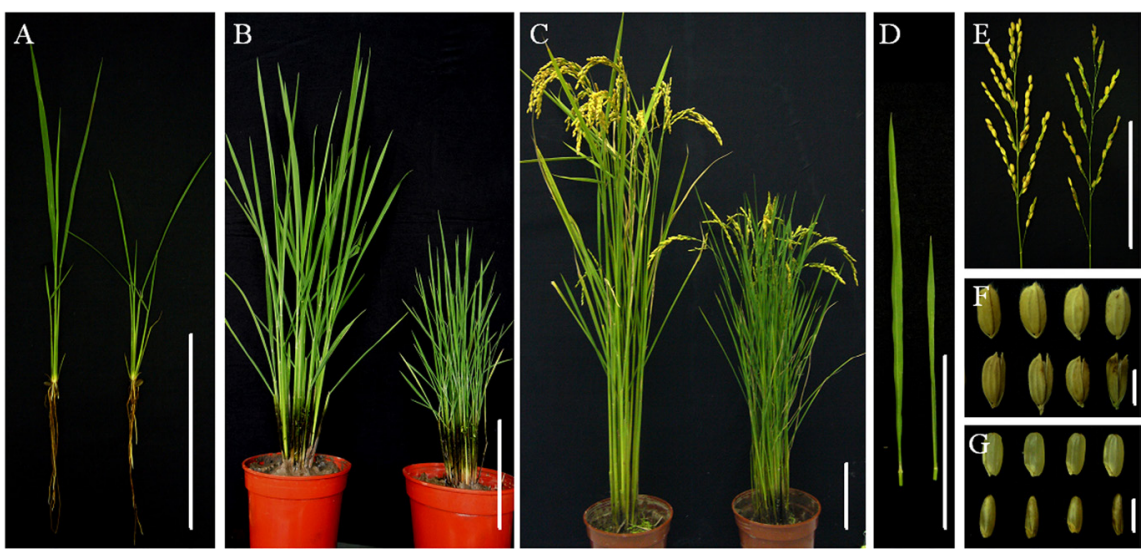

Figure 1. Phenotypic characterization of the $d n l 1$ mutant. A.-C. Wild-type and $d n l 1$ plants 25 days (A), 60 days (B) after sowing and maturity $(\mathbf{C})$; D.-G. flag leaves $(\mathbf{D})$, panicles $(\mathbf{E})$, grains $(\mathbf{F})$, and brown rice $(\mathbf{G})$ of the main panicles of wild-type and $d n l l$ plants. A.-E. The left one is the wild-type and the right one is the $d n l 1$ mutant. F. and G. The above ones are the wild-type and the bottom ones are the $d n l 1$ mutant plants. Bar $=10 \mathrm{~cm}(\mathbf{A} .-\mathbf{E}$.) or $0.5 \mathrm{~cm}$ (F. and G.).

Table 3. Phenotypic analysis of the dnll mutant.
\begin{tabular}{lccccccc}
\hline Lines & $\begin{array}{c}\text { Plant height } \\
(\mathrm{cm})\end{array}$ & Tillers & $\begin{array}{c}\text { Flag leaf width } \\
(\mathrm{cm})\end{array}$ & $\begin{array}{c}\text { Culm diameter } \\
(\mathrm{cm})\end{array}$ & $\begin{array}{c}\text { Seed setting rate } \\
(\%)\end{array}$ & $\begin{array}{c}\text { No. of spikelets } \\
\text { per panicle }\end{array}$ & $\begin{array}{c}\text { Weight of } 1000 \\
\text { grains }(\mathrm{g})\end{array}$ \\
\hline Wild-type & $70.23 \pm 2.12$ & $14.6 \pm 4.20$ & $1.19 \pm 0.07$ & $0.35 \pm 0.04$ & $90.67 \pm 2.35$ & $85.05 \pm 3.25$ & $28.85 \pm 0.24$ \\
dnll & $50.33 \pm 4.80$ & $38.88 \pm 6.51$ & $0.56 \pm 0.06$ & $0.22 \pm 0.02$ & $23.91 \pm 5.78$ & $46.12 \pm 5.06$ & $16.81 \pm 0.09$ \\
P value & $1.74 \times 10^{-7}$ & $1.38 \times 10^{-6}$ & $5.71 \times 10^{-14}$ & $7.69 \times 10^{-7}$ & $6.67 \times 10^{-8}$ & $5.32 \times 10^{-5}$ & $2.21 \times 10^{-5}$ \\
\hline$t$-test $)$ & & & & & & & \\
\hline
\end{tabular}

Values are reported as means \pm SD of 10 biological replicates.

\section{Stem growth was affected in the dnl1 mutant}

During maturity, the average height of $d n l 1$ mutant plants was approximately $71 \%$ of its wild-type (Figures 1B, C, 2A and Table 3). The internode elongation patterns between the dnll mutant and wild-type were compared (Figure $2 \mathrm{~A}$ and $\mathrm{B}$ ), and the data showed that the panicles 
and internodes of the dnll mutant were remarkably shorter than those of its wild-type. The stems of the dnll mutant were thinner than those of wild-type, and stem diameter of the dnll mutant was about $63 \%$ of wild-type (Figure 2A, B and Table 3). To determine whether the dwarf phenotype of the $d n l l$ mutant resulted from defective cell division and/or cell elongation, longitudinal sections of internodes I, II, and III of $d n l l$ mutant culms were compared with its wild-type counterparts. As shown in Figure 2C-G, the cell length of the dnll mutant was not significantly shorter, while the total cell number in the y-axes in internodes I, II, and III of the dnll mutant was significantly less than that of its wild-type. This result indicated that total cell number in the $\mathrm{y}$-axes in internodes was the main reason for the dwarf phenotype of the dnll mutant.
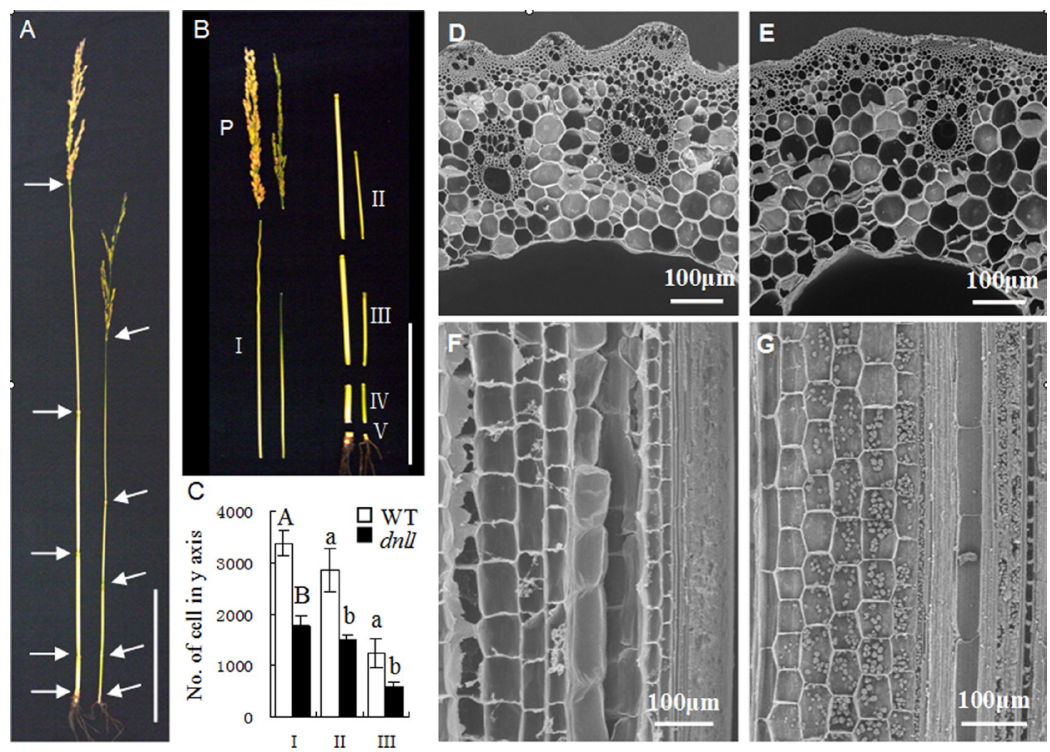

Figure 2. Differences of the culms and scanning electron microscopic (SEM) observation of internodes between wildtype and dnll mutant plants. A. Main culms of wild-type (left) and $d n l 1$ mutant (right) plants. Arrows indicate the positions of nodes. Bar $=15 \mathrm{~cm}$. B. Differences of panicles and internodes of main culms between wild-type (left) and dnll mutant (right) plants. $\mathrm{P}=$ Panicle. Those from I to $\mathrm{V}$ indicate the corresponding internodes from top to bottom. Bar $=15 \mathrm{~cm}$. C. Total cell number of internodes I, II, and III in y-axis. The presence of different letters indicates significance (lowercase and capital letters represent $\mathrm{P}<0.05$ and 0.01 , respectively); D. and $\mathbf{E}$. SEM of transverse sections of the middle part of internode I of wild-type (D) and $d n l l$ mutant (E) plants at the mature stage; F. and $\mathbf{G}$. SEM of longitudinal sections of the middle part of internode III of wild-type (F) and dnll mutant $(\mathbf{G})$ plants at the mature stage.

\section{Genetic analysis and fine mapping of the dnl1 mutant gene}

Plant height, leaf width, stem diameter, and other phenotypes of $F_{1}$ hybrids derived from the cross between the $d n l 1$ mutant and wild-type cv. Asominori, cv. Nanjin 11 were normal. The $\mathrm{F}_{2}$ populations derived from the cross between the dnll mutant and wild-type cv. Asominori, cv. Nanjin11 showed that normal and dwarf, narrow-leaf plants of the $2 \mathrm{~F}_{2}$ populations segregated as 357:141 and 2905:901, respectively $\left(\chi^{2}=2.74,3.50<\chi_{2,0.05}^{2}=3.84, \mathrm{P}>\right.$ $0.05)$, which indicated that the $d n l 1$ mutation was controlled by a single recessive gene. 
The $d n l 1$ gene was primarily mapped between the InDel markers Ind12-11 and SSR marker RM8214 on the short arm of chromosome 12 using 22 dwarf, narrow-leaf plants from the $\mathrm{F}_{2}$ population of a cross between the dnll mutant and cv. Nanjin11 (Figure 3A). The $d n l 1$ gene was then localized between markers Ind12-11 and RM1880, with genetic distances of 3.1 and $5.5 \mathrm{cM}$, respectively, based on 184 mutant plants (Figure 3B). After more InDel markers were developed (Table 1), the $d n l l$ gene was further localized in an interval of $285.75 \mathrm{~kb}$ bracketed by the markers Ind12-2 and Ind12-8, based on 901 mutant individuals (Figure $3 \mathrm{C}$ and D).

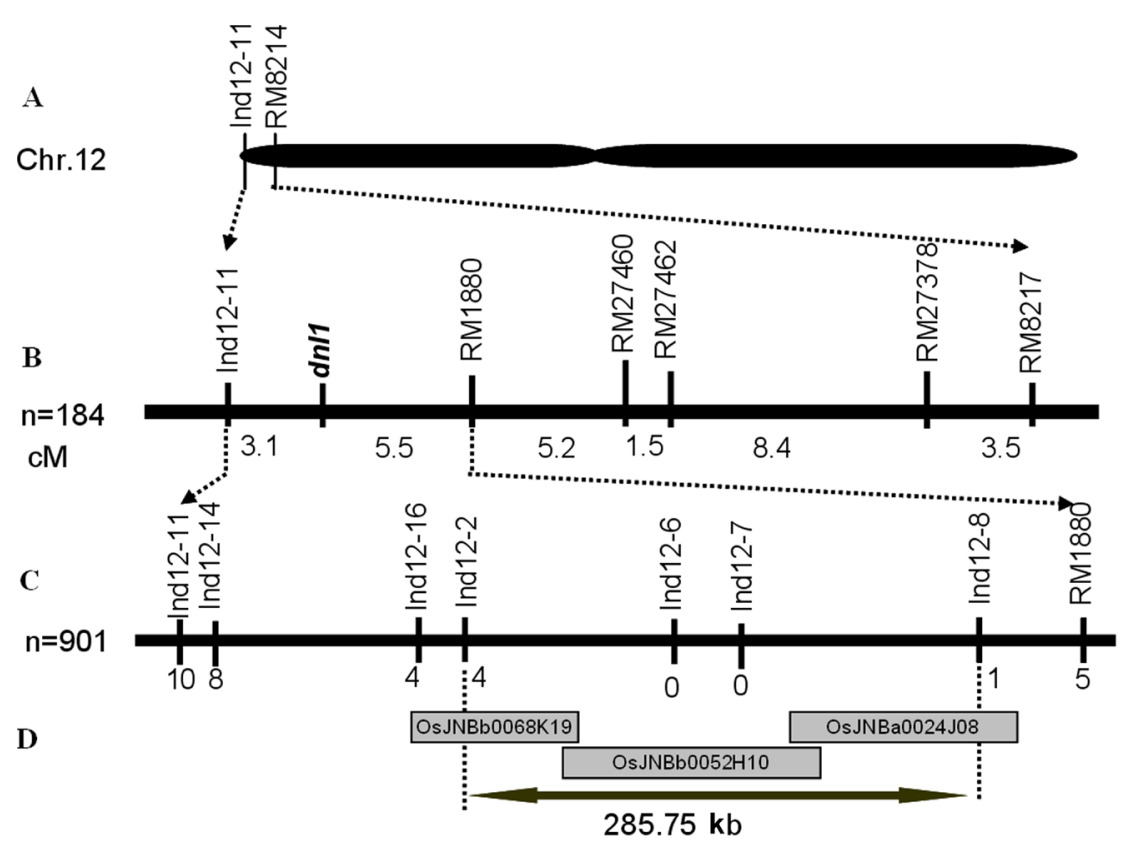

Figure 3. Genetic and physical maps of the $d n l 1$ gene on rice chromosome 12 . A. and B. Primary mapping of the $d n l$ gene; C. fine mapping of the $d n l l$ gene; D. BAC contigs around the $d n l l$ locus.

\section{dnl1 is a GA-insensitive mutant}

Recent molecular genetic approaches have revealed that plant dwarfism is often caused by defects in the biosynthesis and perception of plant hormones such as GAs and brassinosteroids (BRs) (Ueguchi-Tanaka et al., 2000; Sasaki et al., 2002, 2003; Hong et al., 2003; Itoh et al., 2004; Tanabe et al., 2005). To determine whether $d n l l$ is deficient in or insensitive to GA or BR, we treated dnll mutants with GA3 and most bioactive BR compound, BL (Figure 4). When treated with BL, the length of the second leaf sheath of $d n l 1$ mutants was not elongated and was almost the same as that of wild-type plants (Figure 4B). This result indicates that the $d n l 1$ mutant gene has no correlation with BR plant hormones. However, the response of $d n l l$ to exogenously applied GA3 was much lower than that of the wild-type plants (Figure 4A), indicating that the $d n l 1$ mutant has a defect in GA responsiveness. 

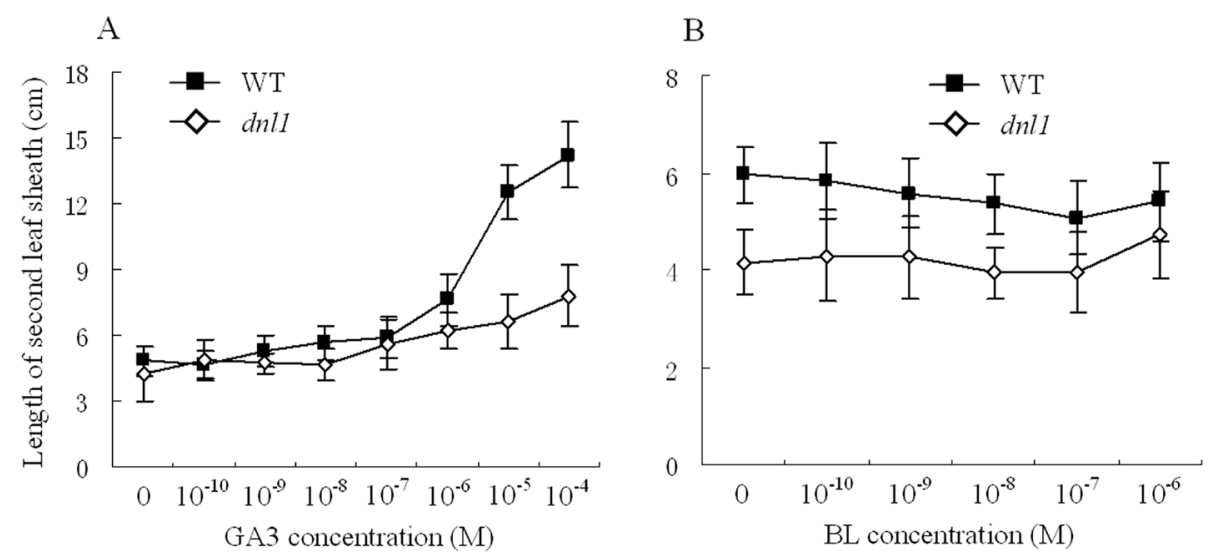

Figure 4. Elongation of second leaf sheath in response to A. gibberellin (GA3) and B. brassinolide (BL) treatment in wild-type (squares) and $d n l l$ mutant (lozenges) plants. Data are reported as means $\pm \mathrm{SD} ; \mathrm{N}=20$.

\section{Expression of genes involved in gibberellin metabolic pathways is affected in the dnll mutant}

The dnll mutant is insensitive to GA3 (Figure 4A), so the expression analysis of genes involved in GA biosynthesis and response pathways in the $d n l 1$ mutant was investigated by real-time PCR (Figure 5). The results showed that the expression of the GA response genes GID1 and EUI1 was not changed, while the expression of the GA response genes D1, SLR1, and GID2 and GA biosynthesis genes GA20ox2, GA20ox3, GA3ox2, GA2ox1, and GA2ox3 significantly differed in the $d n l 1$ mutant. The results suggested that the $D N L 1$ gene likely plays an important role in GA metabolic pathways.

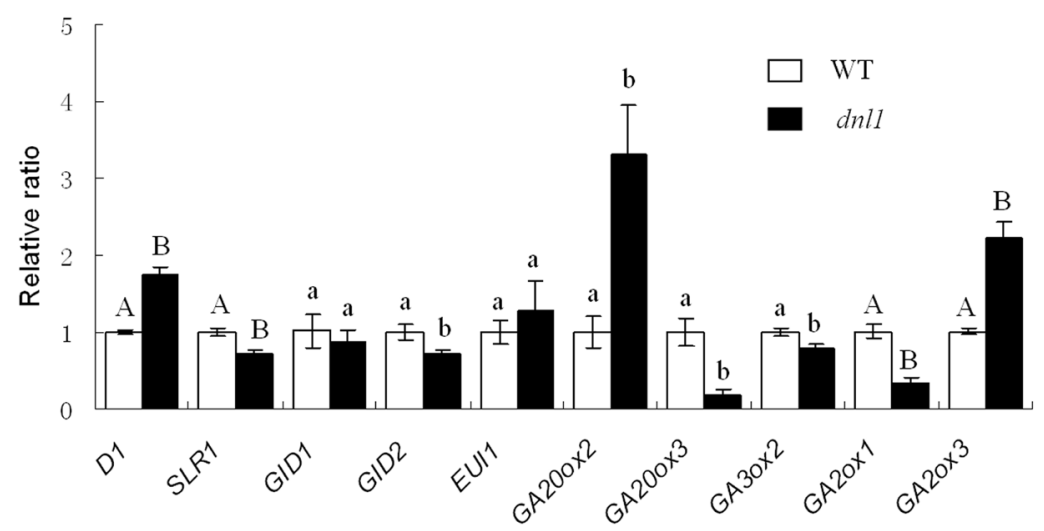

Figure 5. Expression analysis of genes involved in the gibberellin synthesis and response pathway in $d n l 1$ mutant and wild-type plants. Quantitative real-time PCR was performed with total RNA from 40-day-old plants. Transcript levels of all genes tested in the wild-type were arbitrarily set to 1 . Values are reported as means $\pm \mathrm{SD}$ of the 3 replicates. For each gene, the presence of different letters indicates significance (lowercase and capital letters represent $\mathrm{P}<0.05$ and 0.01 , respectively). 


\section{DISCUSSION}

Plant height is one of the most important agronomic traits of rice. The dwarf mutants are ideal materials in research on the mechanism for the regulation of rice plant height. Up to the present, more than 60 rice dwarf mutants have been reported (Matsuo et al., 1997). Besides having a short plant height, many dwarf mutants also have many other abnormal phenotypes, such as small grains, multiple tillers, narrow or rolled leaves (Ueguchi-Tanaka et al., 2000; Tanabe et al., 2005; Arite et al., 2007; Li et al., 2009). On the basis of these associated traits, rice dwarf mutants could be divided into 3 groups, small grain dwarfs, such as $d 1$ and $d 11$, malformation dwarfs, such as $d 2, d 6$, and $t d d 1$, high-tillering dwarf, such as $h t d 1$, htd2, $d 10$, and $d 27$ (Ueguchi-Tanaka et al., 2000; Nagasaki et al., 2001; Hong et al., 2003; Tanabe et al., 2005; Zou et al., 2005; Arite et al., 2007; Liu et al., 2009; Sazuka et al., 2009; Lin et al., 2009). In the present study, besides dwarfism, the $d n l l$ mutant also showed narrow leaf and thin culm characters and higher tiller number, but the number of grains per panicle, the seed setting rate, and the grain weight were significantly decreased compared with wild-type (Figure 1 and Table 3). Earlier, another dwarf mutant $n d 1$ (narrow leaf and dwarf1) with almost the same phenotypes as $d n l l$ had been reported (Li et al., 2009). However, $n d l$ is a GA-sensitive dwarf mutant, and $d n l l$ is not (Figure 4A). The fine mapping of $d n l l$ suggested that they are on different regions of the chromosome (Figure 3). Also, no other dwarf mutants have been reported in the chromosome region of $d n l l$. Therefore, $d n l l$ is a new dwarf and narrow-leaf mutant, and the DNL1 gene may have pleiotropic effects on plant height, yield, and plant architecture in rice. The cloning and function analysis of $D N L 1$ will have important significance for further understanding the molecular mechanism of the regulation of growth and development of rice.

The control of rice plant height is mostly related to the biosynthesis of and responsiveness to phytohormones such as GAs, BL and strigolactone (Ueguchi-Tanaka et al., 2000; Sasaki et al., 2002, 2003; Hong et al., 2003; Itoh et al., 2004; Tanabe et al., 2005; Arite et al., 2007; Lin et al., 2009). A series of genes encoding the enzymes involved in the GA biosynthetic pathway have been cloned from a variety of species (Hedden and Phillips, 2000). In contrast to the rapid progress in the study of GA biosynthesis, much less is known about how plants perceive GA and how the GA signal is transmitted to cause GA-regulated plant growth. When the dwarf mutants were related to the GA responsiveness and signal transduction, they were insensitive to the exogenous GAs (Ikeda et al., 2001; Sasaki et al., 2003; Ueguchi-Tanaka et al., 2000, 2005). Thus, GA-insensitive mutants are ideal research materials to study how plants perceive GA and how the GA signal is transmitted. On the basis of research on the GA-insensitive dwarf mutants $s l r 1$, gidl, and gid2, important progress has been made in GA responsiveness and signal transduction. The GA-GID1-DELLA pathway, which is the basic GA signal transduction pathway, has been established (Ikeda et al., 2001; Sasaki et al., 2003; Ueguchi-Tanaka et al., 2005). When the GA receptor GID1 receives the GA signal, bound to SLR1, a rice DELLA protein, which is a repressor of GA signaling, forms a GA-GID1-SLR1 complex. The SLR1 protein is then degraded through the SCF-mediated 26S proteasome system (Ikeda et al., 2001). The GA signal then regulates plant growth and development. GID2 is the F-box protein subunit of the SCF complex (Sasaki et al., 2003). In this study, the results of phytohormone induction of shoot elongation suggested that $d n l 1$ is a GA-insensitive dwarf mutant (Figure 4A). Therefore, the dnl1 mutant may have a defect in GA responsiveness, and the DNL1 gene should be a regulator in the GA responsiveness and signal transduction path- 
way. The results of expression analysis of genes involved in GA biosynthesis and response pathways showed that the expression of the GA response genes D1, SLR1, and GID2 and GA biosynthesis genes GA20ox2, GA20ox3, GA3ox2, GA2ox1, and GA2ox3 significantly changed in the dnll mutant. These results further suggest that DNL1 is very important in the GA signal transduction pathway. The change in expression of GA biosynthesis genes may be due to the feedback regulation of endogenous GAs.

Our results about the novel GA-insensitive dwarf and narrow-leaf mutant $d n l 1$ are very useful for $D N L 1$ gene cloning and to improve our understanding of the molecular mechanism of the regulation of growth and development of rice. However, cloning of the DNL1 gene and its role in GA metabolic pathways and regulation of growth and development of rice still need further research.

\section{ACKNOWLEDGMENTS}

Research supported by the '863' Programs of China (grant \#2011AA10A101), the National S\&T Major Project (\#2011ZX08001-001, \#2011ZX08001-002, and \#2011ZX08001006), the Central Level, Non-Profit, Scientific Research Institutes Basic R\&D Operations Special Fund (\#2012RG002-1), and the Natural Science Foundation of Zhejiang Province (grant \#LY12C13003).

\section{REFERENCES}

Arite T, Iwata H, Ohshima K, Maekawa M, et al. (2007). DWARF10, an RMS1/MAX4/DAD1 ortholog, controls lateral bud outgrowth in rice. Plant J. 51: 1019-1029.

Davies PJ (1995). Plant Hormones: Physiology, Biochemistry and Molecular Biology. Kluwer Academic Publishers, Dordrecht.

Hargrove TR and Cabanilla VL (1979). The impact of semidwarf varieties on Asian rice breeding programs. BioScience 29: 731-735.

Hedden P and Phillips AL (2000). Gibberellin metabolism: new insights revealed by the genes. Trends Plant Sci. 5: $523-$ 530.

Hong Z, Ueguchi-Tanaka M, Umemura K, Uozu S, et al. (2003). A rice brassinosteroid-deficient mutant, ebisu dwarf(d2), is caused by a loss of function of a new member of cytochrome P450. Plant Cell 15: 2900-2910.

Ikeda A, Ueguchi-Tanaka M, Sonoda Y, Kitano H, et al. (2001). slender rice, a constitutive gibberellin response mutant, is caused by a null mutation of the SLRI gene, an ortholog of the height-regulating gene GAI/RGA/RHT/D8. Plant Cell 13: 999-1010.

Itoh H, Ueguchi-Tanaka M, Sentoku N, Kitano H, et al. (2001). Cloning and functional analysis of two gibberellin $3 \beta$-hydroxylase genes that are differently expressed during the growth of rice. Proc. Natl. Acad. Sci. U. S. A. 98: 8909-8914.

Itoh H, Tatsumi T, Sakamoto T, Otomo K, et al. (2004). A rice semi-dwarf gene, Tan-Ginbozu (D35), encodes the gibberellin biosynthesis enzyme, ent-kaurene oxidase. Plant Mol. Biol. 54: 533-547.

Khush GS (1999). Green revolution: preparing for the 21st century. Genome 42: 646-655.

Li M, Xiong G, Li R, Cui J, et al. (2009). Rice cellulose synthase-like D4 is essential for normal cell-wall biosynthesis and plant growth. Plant J. 60: 1055-1069.

Lin H, Wang R, Qian Q, Yan M, et al. (2009). DWARF27, an iron-containing protein required for the biosynthesis of strigolactones, regulates rice tiller bud outgrowth. Plant Cell 21: 1512-1525.

Liu W, Wu C, Fu Y, Hu G, et al. (2009). Identification and characterization of HTD2: a novel gene negatively regulating tiller bud outgrowth in rice. Planta 230: 649-658.

Matsuo T, Futsuhara Y, Kikuchi F and Yamaguchi H (1997). Science of the Rice Plant. Vol. 3: Genetics, Nobunkyo, Tokyo. 302-303.

Nagasaki H, Sakamoto T, Sato Y and Matsuoka M (2001). Functional analysis of the conserved domains of a rice KNOX homeodomain protein, OSH15. Plant Cell 13: 2085-2098. 
Sakamoto T, Miura K, Itoh H, Tatsumi T, et al. (2004). An overview of gibberellin metabolism enzyme genes and their related mutants in rice. Plant Physiol. 134: 1642-1653.

Sasaki A, Ashikari M, Ueguchi-Tanaka M, Itoh H, et al. (2002). Green revolution: a mutant gibberellin-synthesis gene in rice. Nature 416: 701-702.

Sasaki A, Itoh H, Gomi K, Ueguchi-Tanaka M, et al. (2003). Accumulation of phosphorylated repressor for gibberellin signaling in an F-box mutant. Science 299: 1896-1898.

Sazuka T, Kamiya N, Nishimura T, Ohmae K, et al. (2009). A rice tryptophan deficient dwarf mutant, tdd1, contains a reduced level of indole acetic acid and develops abnormal flowers and organless embryos. Plant J. 60: 227-241.

Tanabe S, Ashikari M, Fujioka S, Takatsuto S, et al. (2005). A novel cytochrome P450 is implicated in brassinosteroid biosynthesis via the characterization of a rice dwarf mutant, dwarf11, with reduced seed length. Plant Cell 17: 776790.

Ueguchi-Tanaka M, Fujisawa Y, Kobayashi M, Ashikari M, et al. (2000). Rice dwarf mutant $d 1$, which is defective in the $\alpha$ subunit of the heterotrimeric $\mathrm{G}$ protein, affects gibberellin signal transduction. Proc. Natl. Acad. Sci. U. S. A. 97: 11638-11643.

Ueguchi-Tanaka M, Ashikari M, Nakajima M, Itoh $\mathrm{H}$, et al. (2005). GIBBERELLIN INSENSITIVE DWARF1 encodes a soluble receptor for gibberellin. Nature 437: 693-698.

Zhang QF, Shen BZ, Dai XK, Mei MH, et al. (1994). Using bulked extremes and recessive class to map genes for photoperiod-sensitive genic male sterility in rice. Proc. Natl. Acad. Sci. U. S. A. 91: 8675-8679.

Zou J, Chen Z, Zhang S, Zhang W, et al. (2005). Characterizations and fine mapping of a mutant gene for high tillering and dwarf in rice (Oryza sativa L.). Planta 222: 604-612. 